Published By Institute of Physics Publishing for SISSA/ISAS

RECEIVED: December 2, 2003

ACCEPTED: January 30, 2004

\title{
Inflationary perturbations from deformed CFT
}

\author{
Jan Pieter van der Schaar \\ CERN Theory Division \\ CH-1211 Geneva 23, Switzerland \\ E-mail: Jan.Pieter.van.der.Schaar@cern.ch
}

\begin{abstract}
We present a new method to calculate the spectrum of (slow-roll) inflationary perturbations, inspired by the conjectured dS/CFT correspondence. We show how the standard result for the spectrum of inflationary perturbations can be obtained from deformed CFT correlators, whose behavior is determined by the Callan-Symanzik equation. We discuss the possible advantages of this approach and end with some comments on the role of holography in $\mathrm{dS} / \mathrm{CFT}$ and its relation to the universal nature of the spectrum of inflationary perturbations.
\end{abstract}

Keywords: AdS-CFT and dS-CFT Correspondence, Cosmology of Theories beyond the SM. 


\section{Contents}

1. Introduction

2. dS/CFT and holographic RG-flow 3

2.1 Some dS/CFT preliminaries

2.2 Homogeneous monotonic flow and its holographic description

3. Inflationary perturbations 8

3.1 De Sitter scalar perturbations 8

3.2 The CMB power spectrum and spectral indices 11

4. Inflationary perturbations from CFT correlators 14

4.1 Relating correlation functions to expectation values 15

4.2 CFT operators, energy momentum and inflationary perturbations 16

4.3 RG-flow equations and the spectrum of inflationary perturbations 17

5. Holography and universality of inflationary perturbations 20

6. Note added 21

\section{Introduction}

Observational evidence has completely revised our ideas on the history and future fate of our universe. As long suspected and recently shown to be in excellent agreement with the Cosmic Microwave Background (CMB) observations [1], the early universe probably experienced a brief phase of slow-roll inflation, corresponding to accelerated expansion close to a de Sitter spacetime. More surprisingly, supernovae observations [2] suggest that our universe is currently also experiencing a phase of accelerated expansion, presumably again described by an approximate de Sitter phase. These phenomenological considerations partly explain the current surge of theoretical interest in de Sitter spacetime. On the other hand there have always been strong theoretical motivations to study de Sitter space. In particular, since de Sitter spacetime can be considered the most symmetric cosmological equivalent of a black hole, it seems to be a nice arena to study some of the confusing properties having to do with the presence of event horizons.

String theory has made some progress in understanding certain aspects of black hole physics. An extremely powerful idea that seems to be concretely realized in string theory is the holographic principle [3]. In the context of Anti-de Sitter gravity the holographic principle is realized through the AdS/CFT correspondence [4, 5. In a leap of faith attempts 
have been made to generalize the AdS/CFT correspondence to apply it in the context of de Sitter space, leading to the dS/CFT conjecture [6]. Just as the radial coordinate in AdS space is related to the scale parameter in the dual lorentzian CFT, in dS/CFT the timelike coordinate of de Sitter space should be related to the scale parameter in an euclidean CFT. This also suggests that (homogeneous) departures from AdS or dS will correspond to renormalization group (RG) flows in the corresponding deformed CFT's. However, the absence of supersymmetry, a concrete string theory example and more fundamental objections [7], raise severe doubts on the correctness and usefulness of the dS/CFT proposal. Nevertheless, from a pragmatic point of view it seems that some problems in four-dimensional de Sitter space can certainly be addressed using a general three-dimensional CFT description, as conjectured by the dS/CFT correspondence but possibly just a result of the symmetries of the de Sitter background. From that more modest point of view a CFT approach would just correspond to a different perspective on some problems in (approximate) de Sitter space, and the CFT approach would in principle not be able to teach us anything beyond what was known already. Nevertheless we think this could still be an interesting and worthwhile enterprise, hopefully giving us at least some qualitative hints on some of the properties of quantum gravity in de Sitter spacetime.

As already mentioned, an important point is that homogeneous deformations away from de Sitter spacetime, in general corresponding to Friedmann-Robertson-LemaitreWalker (FRLW) cosmologies but for small deformations giving rise to inflationary cosmologies, are interpreted as departures from perfect scale invariance in the euclidean CFT. This would then give rise to renormalization group flow away from the conformal fixed point [13, 14], a situation that has been particularly well-studied and understood in the AdS/CFT context [8]-[12]. Since approximate de Sitter spaces seem to play an important role in the history of our universe, a holographic RG-flow description could have direct phenomenological applications. In particular the early inflationary phase and its production of small perturbations that are the primordial seeds of the observed CMB, and therefore of large scale structures in our universe, would be interesting examples to study from a CFT point of view and is the focus of this article. One strong reason to suspect that this should be possible is the approximate scale invariant nature of the primordial spectrum of density perturbations [15, 16, 17]. This possibility was first anticipated in [18], and shown to work for massless perturbations in [19]. We will describe the more general (massive) case and make full use of the holographic RG-equations, that for the de Sitter case were only determined in all detail very recently [20]. As we will explain such an approach sheds new light on the universal nature of the spectrum of inflationary perturbations and the related question of the effect of trans-Planckian physics on the primordial CMB spectrum, a subject that has attracted a lot of attention recently [21]. We stress that the dS/CFT correspondence is not probing the static patch describing a single bulk Hubble volume and discuss the meaning of holography in $\mathrm{dS} / \mathrm{CFT}$. We suggest that the main implication of holography in cases where the holographic coordinate is timelike should be universality.

The structure of this paper is as follows. In section 2 we briefly review the dS/CFT correspondence and the interpretation of broken scale invariance in terms of holographic RG-flow. We then move on to present a minimal review of the calculation of the spectrum 
of inflationary density perturbations. After that we show that the spectrum of inflationary perturbations can be derived starting from CFT correlators and introducing small deformations satisfying the Callan-Symanzik equation. We devote a separate section to a discussion on the holographic interpretation of the primordial CMB calculation in terms of CFT correlators. There we also discuss the relation between the deformed CFT approach and the universal nature of the spectrum of inflationary perturbations.

\section{2. dS/CFT and holographic RG-flow}

We give a short review of $\mathrm{dS} / \mathrm{CFT}$ and the holographic correspondence between cosmological evolution and renormalization group flow. For similar, more detailed discussions we refer to [18, 22, 23].

\subsection{Some dS/CFT preliminaries}

De Sitter space can be obtained from Anti-de Sitter by performing a so-called double Wick rotation. Starting from the AdS metric in stereographic coordinates one performs the following transformations

$$
\tau \rightarrow i x_{d-1}, \quad \lambda \rightarrow-i t, \quad h \rightarrow i H,
$$

to obtain de Sitter space in planar, or inflationary, coordinates (covering only half of de Sitter space)

$$
d s^{2}=-d t^{2}+e^{2 H t} d x_{d}^{2} .
$$

Introducing the scale factor $a=e^{H t}$ and defining the following coordinate

$$
\mu=H a,
$$

which has the dimension of energy, we obtain the following parametrization of $(d+1)$ dimensional de Sitter

$$
d s^{2}=H^{-2}\left[-\mu^{-2} d \mu^{2}+\mu^{2} d x_{d}^{2}\right] .
$$

In dS/CFT the parameter $\mu$ will again have the natural interpretation of the energy scale in a dual CFT description. The boundaries of the AdS and dS metrics in this "holographic" parametrization are obtained in the limit $\mu=u \rightarrow \infty$. We also note that for de Sitter this parametrization is related to a de Sitter parametrization using conformal time $\eta$, which is equal to $\eta= \pm 1 / \mu$ and therefore naturally interpreted as the length scale in a dual field theory. This corresponds to the de Sitter analogue of the Poincaré coordinates in AdS. De Sitter in conformal time gives rise to the conformally flat metric

$$
d s^{2}=(H \eta)^{-2}\left[-d \eta^{2}+d x_{d}^{2}\right],
$$

where the infinite future corresponds to $\eta=0$ and the infinite past to $\eta \rightarrow \infty$.

As in AdS/CFT we would now like to introduce perturbations and the easiest thing to consider is adding scalar fields to de Sitter. As $t \rightarrow \infty$ the homogeneous part of the scalar

field is dominant, giving us the following asymptotic behavior of the metric and scalar field

$$
d s^{2} \sim-d t^{2}+e^{2 H t} \hat{g}_{i j} d x^{i} d x^{j}
$$




$$
\phi \sim e^{\lambda H t} \hat{\phi}
$$

where $\lambda_{ \pm}=-d / 2 \pm \sqrt{d^{2} / 4-m^{2} / H^{2}}$ [6]. Scale invariance demands that under a time translation $H t \rightarrow H t+\delta$ and $x_{i} \rightarrow\left(e^{\delta}\right)^{-1} x_{i}$, the full asymptotic solution remains invariant. This is ensured if the ("boundary" ${ }^{1}$ ) fields $\hat{g}_{i j}$ and $\hat{\phi}$ transform as follows

$$
\hat{g}_{i j} \rightarrow\left(e^{\delta}\right)^{0} \hat{g}_{i j}, \quad \hat{\phi} \rightarrow\left(e^{\delta}\right)^{-\lambda} \hat{\phi} .
$$

In a holographic dual field theory description these boundary fields are supposed to be sources of dual operators in the field theory. The metric boundary field $\hat{g}_{i j}$ is the natural source of the energy momentum tensor in a dual field theory

$$
\mathcal{S}_{\hat{g}} \propto \int d^{d} x \hat{g}_{i j} \mathcal{T}^{i j}
$$

whereas the scalar boundary fields should correspond to sources of (gauge invariant) operators in a holographic dual

$$
\mathcal{S}_{\hat{\phi}} \propto \int d^{d} x \hat{\phi} \mathcal{O}
$$

From the scaling transformations of the boundary fields and the total scale invariance of the holographically dual lagrangian we therefore find the following scaling dimensions $\mathcal{O} \sim\left(e^{\delta}\right)^{\Delta} \mathcal{O}$ of the dual operators

$$
\Delta_{\mathcal{T}_{i j}}=d, \quad \Delta_{\mathcal{O}}=\lambda+d .
$$

In case one is interested in (homogeneous) deformations of the CFT, typically breaking conformal invariance and leading to RG-flow, the behavior is determined by the scaling dimension of the operator deforming the theory. To remind ourselves: marginal operators correspond to scaling dimensions equal to $d$, so indeed the energy momentum tensor corresponds to a marginal operator, as it should. Scaling dimensions $\Delta>d$ correspond to irrelevant non-renormalizable perturbations, whereas operators with $\Delta<d$ are relevant normalizable operators. Relevant operators will take us away from the UV fixed point.

Formally the AdS/CFT correspondence can be summarized by the mathematical statement that the partition function of a dual CFT is given by the (semi-classical) wavefunction of quantum gravity on AdS space [24]

$$
\mathcal{Z}_{\mathrm{CFT}}=\Psi_{A d S} \approx e^{i S_{A d S}} .
$$

To make sense out of this expression one typically Wick rotates to euclidean AdS, giving $\exp -S_{E A d S}$ for the euclidean partition function. To explicitly relate boundary correlation functions to bulk quantities we treat the boundary fields $\hat{\phi}$ as sources for CFT operators and, as usual, functionally differentiate with respect to the sources, setting the sources to zero afterward. From the bulk perspective this means that in the semiclassical limit

\footnotetext{
${ }^{1}$ The "boundary" is defined as the spacelike section at $t \rightarrow \infty$.
} 
we evaluate the bulk action on a (normalizable) solution to the equations of motion with suitable boundary conditions giving the dependence of the bulk action on $\hat{\phi}$.

$$
\left\langle\mathcal{O}_{1} \ldots \mathcal{O}_{n}\right\rangle=\left.\frac{\delta^{n} \mathcal{Z}_{\mathrm{CFT}}}{\delta \hat{\phi}_{1} \ldots \delta \hat{\phi}_{n}}\right|_{\hat{\phi}=0}=\left.\frac{\delta^{n} \Psi_{\operatorname{AdS}}[\hat{\phi}]}{\delta \hat{\phi}_{1} \ldots \delta \hat{\phi}_{n}}\right|_{\hat{\phi}=0},
$$

where $\bar{\phi}$ is the source of the CFT operator $\mathcal{O}$. Any singularities that arise in the action should be treated just as if one is renormalizing a field theory and will typically lead to (holographic) RG-flow [11, 12]. As already alluded to this RG-flow can be understood as being described by a deformation with a local operator

$$
S_{\mathrm{CFT}} \rightarrow S_{\mathrm{CFT}}+g \int d^{d} x \mathcal{O}(x),
$$

which by means of the correspondence (2.9) suggests a relation to homogeneous bulk gravitational solutions $\phi \sim g$. Deforming the CFT with an operator of dimension $\Delta$ implies the lagrangian deformation scales as $\Delta-d=\lambda(2.10)$. This coincides with the asymptotic behavior of (homogeneous) scalar fields (2.6). Relevant deformations vanish in the UV to be consistent with the presence of a conformal fixed point, whereas irrelevant deformations blow up in the UV. From the bulk perspective this is just saying that asymptotic de Sitter space is only consistent with decaying behavior of the (homogeneous) scalar field. When the scalar field blows up de Sitter space is not the asymptotic solution.

Moving from AdS to dS we will assume that the same recipe applies for dS/CFT, i.e.

$$
\mathcal{Z}_{\mathrm{CFT}}=\Psi_{d S} \approx e^{i S_{d S}}
$$

where the CFT in this case is already defined in euclidean space and the right hand side corresponds to the wavefunction of quantum gravity in de Sitter space, which can now honestly be interpreted as the "wavefunction of the universe". As in AdS the de Sitter action will generically diverge when approaching the "boundary" in the infinite future. Just as in field theory, the (local) divergences can be canceled by counterterms leading to a renormalized action satisfying RG equations. For de Sitter this holographic renormalization procedure has only recently been carefully worked out [20].

\subsection{Homogeneous monotonic flow and its holographic description}

Consider $(d+1)$-dimensional Einstein gravity coupled to scalars $\phi^{I}$ via

$$
\mathcal{L}=\frac{1}{2 \kappa^{2}} R-\frac{1}{2} G_{I J} \partial_{\mu} \phi^{I} \partial^{\mu} \phi^{J}-V\left(\phi^{I}\right)
$$

where $\kappa^{2}=8 \pi G=1 / M_{p}{ }^{(d-1)}$ and $G_{I J}$ is the metric on the moduli space of the scalars $\phi^{I}$, which we will assume to be independent of the scalars $\phi^{I}$ for the time being. It could perhaps be interesting to relax this assumption which would involve introducing covariant derivatives with respect to the moduli space metric. 
The potential $V\left(\phi^{I}\right)$ is assumed to have de Sitter extrema. Using the standard spatially flat FRW metric

$$
d s^{2}=-d t^{2}+a(t)^{2} d x_{i} d x^{i}
$$

with $i$ running from 1 to $d$ and assuming spatial isotropy $\vec{\nabla} \phi^{I}=0$, the equations of motion are given by

$$
\begin{aligned}
\ddot{\phi}^{I}+d H \dot{\phi}^{I}+G^{I J} \frac{d V}{d \phi^{J}} & =0, \\
H^{2} & =\frac{2}{d(d-1)} \kappa^{2} \rho, \\
\dot{H} & =-\frac{1}{(d-1)} \kappa^{2}(\rho+p),
\end{aligned}
$$

where the Hubble parameter $H$ is $H \equiv \dot{a} / a$, the dot representing a derivative with respect to the coordinate time $t$, and the density $\rho$ and pressure $p$ are given by

$$
\begin{aligned}
& \rho=\frac{1}{2} G_{I J} \dot{\phi}^{I} \dot{\phi}^{J}+V\left(\phi^{I}\right), \\
& p=\frac{1}{2} G_{I J} \dot{\phi}^{I} \dot{\phi}^{J}-V\left(\phi^{I}\right) .
\end{aligned}
$$

The equations of motion give

$$
d(\omega+1)=-\frac{2 \dot{H}}{H^{2}}
$$

for the equation of state parameter $\omega \equiv p / \rho$. Also recall that one of the equations of motion is redundant, e.g. (2.17) and (2.18) imply (2.19). For completeness let us also separately give the expression for the Ricci scalar $R$

$$
R \equiv g_{\mu \nu} R^{\mu \nu}=\frac{2 \kappa^{2}}{d-1}(\rho-d p)=2 \kappa^{2}\left[\left(\frac{d+1}{d-1}\right) V\left(\phi^{I}\right)-\frac{1}{2} G_{I J} \dot{\phi}^{I} \dot{\phi}^{J}\right] .
$$

Note that for constant fields $\phi^{I}$ we find

$$
R=\left(\frac{d+1}{d-1}\right) 2 \kappa^{2} V\left(\phi^{I}\right)=d(d+1) H^{2}=\left(\frac{d+1}{d-1}\right) \Lambda
$$

where we used that $\Lambda \equiv 2 \kappa^{2} V\left(\phi^{I}\right)$.

From now on we will restrict ourselves to four bulk spacetime dimensions $(d=3)$ because we will be interested in calculating the primordial CMB spectrum. We will also restrict our attention to a single scalar. The multi-scalar generalization should be rather straightforward. From the previous section it should be clear that we can interpret the scale factor $a$ as proportional to the (deformed) CFT scale parameter $\mu \propto a(2.3)$ and every scalar $\phi$ as a coupling constant $g=\kappa \phi$ deforming the CFT (2.9). This means we can naturally define the beta functions

$$
\beta \equiv \frac{d g}{d \log \mu}=\frac{\kappa}{H} \dot{\phi}
$$


which by virtue of the FRW equation (2.19) have to satisfy

$$
\beta\left(\beta+\frac{2}{\kappa H} \frac{d H}{d \phi}\right)=0 .
$$

Under the natural assumption of monotonic scalar field flow we derive

$$
\beta=-\frac{2}{\kappa H} \frac{d H}{d \phi}
$$

Monotonicity implies that we will not consider scalar field flows that are oscillating in time. This means we will only consider masses $m^{2}<H^{2}$, which is the relevant regime anyway for the spectrum of inflationary perturbations that we will be interested in.

Under this monotonicity assumption we can relate the equation of state parameter (2.21) and the beta function (2.26) as follows

$$
3(\omega+1)=\frac{4}{\kappa^{2}} H^{-2}\left(\frac{d H}{d \phi}\right)^{2}=\beta^{2} .
$$

Recall that for constant equations of state, i.e. matter $(\omega=0)$ or radiation $(\omega=1 / 3)$, the dependence of the density on the scale factor would be $\rho \propto a^{-3(\omega+1)}=a^{-\beta^{2}}$. To decide whether the expansion of the universe is accelerating or decelerating we calculate

$$
\frac{\ddot{a}}{a}=\dot{H}+H^{2}=H^{2}\left(1-\frac{1}{2} \beta^{2}\right)
$$

so whenever $\beta^{2}<2(\omega<-1 / 3)$ the expansion of the universe is accelerating.

Let us also define a function $\lambda$ that will reproduce the conformal scaling dimensions, derived as follows from the beta-functions

$$
\lambda \equiv \frac{1}{\kappa} \frac{d \beta}{d \phi} .
$$

This implies that near a zero of $\beta$ we can approximately write

$$
\lambda=\left.\frac{1}{\kappa} \frac{d \beta}{d \phi}\right|_{\beta=0} \approx-\left.\frac{2}{\kappa^{2} H} \frac{d^{2} H}{d \phi d \phi}\right|_{\beta=0} .
$$

One can show that near a zero of $\beta$, so the extrema of the potential, one can relate this expression to the mass ${ }^{2}$ in the following way

$$
m^{2}=-H^{2}\left(3 \lambda+\lambda^{2}\right)
$$

which just reduces to the standard equations relating scaling dimensions to masses.

For completeness let us also introduce the conjectured holographic c-function 13 given by

$$
c \equiv \frac{1}{\kappa^{2} H^{2}}
$$


and is strictly decreasing from the UV to the IR fixed point because $\dot{H}<0$ when the Null Energy Condition is satisfied $\omega \geq-1$. One can relate the derivative of the holographic c-function with respect to the scalar fields to the beta-function as follows

$$
\frac{d \ln c}{\kappa d \phi}=\beta
$$

Instead taking the derivative with respect to the logarithmic energy scale (the logarithm of the cosmological scale factor $a$, which is related to the number of e-folds $N$ ) we obtain

$$
\frac{d \ln c}{d \ln a}=\frac{d \ln c}{d N}=\beta^{2} .
$$

So this implies we can write

$$
c\left(N_{f}\right)=c\left(N_{i}\right) \exp \int_{N_{i}}^{N_{f}} \beta^{2} d N
$$

Some of these expressions will turn out to be useful later. Note that the entropy in (the static or causal patch of) de Sitter space is given by the area of a single Hubble volume, which is proportional to the holographic c-function

$$
S=\frac{\text { Area }}{4 \pi G}=\frac{2 \pi}{\kappa^{2} H^{2}}=2 \pi c .
$$

After these preliminaries we are now ready to move on to discuss (inhomogeneous) perturbations in de Sitter space.

\section{Inflationary perturbations}

We will present a rather minimal review of inflationary (density) perturbations, concentrating on the final result. More thorough introductions can be found in many places, we would especially like to mention [16, 17, 19]. In particular, bulk approaches based on a Hamilton-Jacobi setup [25, 11, 20] make the appearance of the holographic RG-equations and the possibility to derive the inflationary perturbations from deformed CFT correlators very apparent and natural.

\subsection{De Sitter scalar perturbations}

Abandoning homogeneity of the scalar field and neglecting gravitational back reaction one should solve the following equation

$$
\ddot{\delta \phi}+3 H \dot{\delta \phi}-\frac{1}{a^{2}} \vec{\nabla}^{2} \delta \phi+\frac{d V}{d \delta \phi}=0
$$

We will be expanding around an extremum of the potential, so we can approximate $\frac{d V}{d \phi} \approx$ $m^{2} \delta \phi$. To comply with the standard literature we define conformal time $\eta$ and redefine the scalar field as follows

$$
\eta \equiv-\frac{1}{H a}=-\frac{1}{\dot{a}}, \quad \varphi \equiv a \delta \phi
$$


where $a$ is the scale factor and $H=\frac{\dot{a}}{a}$. Note that the conformal time coordinate equals $1 / \mu$, where $\mu$ can be interpreted as the natural scale parameter in a dual CFT. This enables us to write the scalar equation as

$$
\varphi^{\prime \prime}+\left(\frac{m^{2}}{H^{2}}-2\right) \frac{1}{\eta^{2}} \varphi-\vec{\nabla}^{2} \varphi=0,
$$

where double primes denote the second derivative with respect to the conformal time $\eta$. Fourier expanding in the spatial directions

$$
\varphi(\eta, \vec{x})=\int d^{3} k \varphi_{k}(\eta) e^{i \vec{k} \cdot \vec{x}}
$$

we finally obtain the following equation for the time dependence of the Fourier components $\varphi(\eta)$

$$
\varphi_{k}^{\prime \prime}+\left(k^{2}+\frac{m^{2} / H^{2}-2}{\eta^{2}}\right) \varphi_{k}=0 .
$$

When $m^{2}=0$ this equation is relatively easy to solve and we get

$$
\varphi_{k} \propto\left(1 \pm \frac{i}{\sqrt{k^{2}} \eta}\right) e^{ \pm i \sqrt{k^{2}} \eta}
$$

For arbitrary $m^{2}$ it is more convenient to first proceed as follows. Define $\chi \equiv k \eta=k / \mu$ and $\sqrt{\chi} \psi(\chi) \equiv \varphi(\chi)$. This transforms equation (3.5) into

$$
\psi_{k}^{\prime \prime}+\frac{1}{\chi} \psi_{k}^{\prime}+\left(1-\frac{n^{2}}{\chi^{2}}\right) \psi_{k}=0
$$

where the primes now stand for derivatives with respect to $\chi$ and $n^{2} \equiv \frac{9}{4}-\frac{m^{2}}{H^{2}}$. This is recognized as a Bessel equation with non-integer coefficient $n^{2}$. Note that for $m^{2}<9 H^{2} / 4$ the coefficient $n$ is real, whereas for $m^{2}>9 H^{2} / 4$ it is imaginary. This Bessel equation is solved by the series expansion

$$
\psi_{k}(\chi)=\chi^{n}\left[1-\frac{1}{n+1}\left(\frac{\chi}{2}\right)^{2}+\frac{1}{(n+1)(n+2)} \frac{1}{2 !}\left(\frac{\chi}{2}\right)^{4}-\cdots\right] .
$$

Defining the Bessel functions as

$$
\mathcal{J}_{n}(\chi)=\sum_{r=0}^{\infty} \frac{(-1)^{r}}{r ! \Gamma(n+r+1)}\left(\frac{\chi}{2}\right)^{n+2 r}
$$

and the solution would read $\psi(\chi)=n ! 2^{n} \mathcal{J}_{n}(x)$. Note that for half-integral $n$ the Bessel functions can be simply expressed in terms of trigonometric functions. Starting from $\mathcal{J}_{1 / 2}(\chi)=(2 / \pi \chi)^{1 / 2} \sin \chi$ and $\mathcal{J}_{-1 / 2}(\chi)=(2 / \pi \chi)^{1 / 2} \cos \chi$ one can use recursion relations to show that $\psi_{k}(\chi)=\sqrt{\frac{2}{\pi \chi}}\left(1 \pm \frac{i}{\chi}\right) \exp \pm i \chi$, reproducing the solution for $m^{2}=0(n= \pm 3 / 2)$.

Introducing a second independent solution to (3.7)

$$
\mathcal{Y}_{n}=\frac{\cos n \pi \mathcal{J}_{n}-\mathcal{J}_{-n}}{\sin n \pi}
$$


we can construct the independent solutions known as the Hankel functions

$$
\begin{aligned}
& \mathcal{H}_{n}^{(1)}=\mathcal{J}_{n}+i \mathcal{Y}_{n} \\
& \mathcal{H}_{n}^{(2)}=\mathcal{J}_{n}-i \mathcal{Y}_{n}
\end{aligned}
$$

The most general solution can then be written in terms of the Hankel functions

$$
\psi_{k}(\chi)=A(k) \mathcal{H}_{n}^{(1)}(\chi)+B(k) \mathcal{H}_{n}^{(2)}(\chi)
$$

where $A(k)$ and $B(k)$ are arbitrary constants determined by the boundary and normalization conditions. Normalization should be carried out with respect to the standard (conserved) Klein-Gordon inner product

$$
i \int d \vec{x} \sqrt{\operatorname{det} g_{i j}} \phi(\vec{x}, t)^{\star} \overleftrightarrow{\partial_{t}} \phi(\vec{x}, t)=1
$$

which in momentum space reduces to the following Wronskian

$$
(-i)|k| \chi\left[\psi_{k}^{\star} \partial_{\chi} \psi_{k}-\left(\partial_{\chi} \psi_{k}^{\star}\right) \psi_{k}\right]=1
$$

To determine the constant $A(k)$ that normalizes the solution it is convenient to first determine the asymptotic behaviour as $|\chi| \gg 1$ of the Hankel functions, which up to constant phases behave as follows

$$
\begin{aligned}
& \mathcal{H}_{n}^{(1)}(\chi) \sim \sqrt{\frac{2}{\pi \chi}} e^{i \chi}, \\
& \mathcal{H}_{n}^{(2)}(\chi) \sim \sqrt{\frac{2}{\pi \chi}} e^{-i \chi} .
\end{aligned}
$$

Note that $|\chi| \gg 1$ corresponds to $a \ll 1$, so it corresponds to the scalar field behaviour in the far past of de Sitter space. The limiting behaviour in the far future of de Sitter space corresponds to taking the limit $|\chi| \ll 1$, which in terms of the natural holographic parameter $\mu$ corresponds to $k \ll \mu$, and immediately follows from the expansion (3.8)

$$
\lim _{|\chi| \rightarrow 0} \psi_{k}(\chi) \sim \chi^{n}
$$

which indeed corresponds to the expected scaling behaviour of the scalar field in the far future. We observe that the solutions start out as ordinary oscillating plane waves but at some point stop oscillating (if $n$ is real) with slowly decaying, almost constant, amplitudes. This means that quantum fluctuations, starting out as ordinary plane waves, will at some point stop oscillating with their amplitudes almost frozen. This is the mechanism for the production of classical, stochastic, scalar fluctuations in de Sitter space. Returning to the normalization of the solutions, consider the solution $\psi_{k}(\chi)=A(k) \mathcal{H}_{n}^{(1)}(\chi)$ in the limit $|\chi| \gg 1$, using expression (3.16), and substitute it into (3.15). Note that we have imposed a boundary condition by setting $B(k)=0$, which is the natural choice from the QFT point of view, where this would correspond to selecting the standard euclidean, Bunch-Davies 
vacuum. Other choices have recently attracted a lot of attention because of their potential connection to trans-Planckian physics [30]. Proceeding, one then finds for $A(k)$

$$
A(k)= \pm \sqrt{\frac{\pi}{4 k}}
$$

For later purposes it is useful to determine the $H, a$ and $k$-dependence of the solutions in the limit $|\chi| \ll 1$ (or $a \gg k / H)$. Using (3.18) and realizing that the variable $\chi=-k / H a$ we find for the $H, a$ and $k$ dependence

$$
\delta \phi_{k}=i \frac{H}{k} \chi^{\frac{3}{2}} A(k) \psi_{k} \propto H^{-\left(n+\frac{1}{2}\right)} k^{n} a^{-\left(n+\frac{3}{2}\right)} .
$$

The dominant behavior when $a \gg k / H(\chi \ll 1)$ of a general solution will be given by the $n_{-}=-\sqrt{\frac{9}{4}-\frac{m^{2}}{H^{2}}}$ mode. Because $n_{-}=-3 / 2-\lambda_{+}$we find the following dominant (scaling) behavior as $a \gg k / H$ in terms of $\lambda_{+}$

$$
\lim _{a \rightarrow \infty} \delta \phi_{k} \propto H^{\lambda_{+}+1} k^{-\frac{3}{2}-\lambda_{+}} a^{\lambda_{+}} .
$$

From this expression it is clear that the amplitude of the mode will not change much as time evolves from the time the mode is "frozen", at least as long as $\left|\lambda_{+}\right| \ll 1$ implying $m^{2} \ll H^{2}$. This moment can be estimated to be around $|\chi|=1$ or when $a=a_{*}=k / H$, when the physical wave number equals the Hubble parameter.

\subsection{The CMB power spectrum and spectral indices}

One can view the spectrum of scalar field fluctuations in (pure) de Sitter space as the source for scalar curvature perturbations during inflation. The mean square scalar field fluctuations in Fourier space are given by $\left|\delta \phi_{k}\right|^{2}$. The power spectrum can be written as

$$
\mathcal{P}_{\delta \phi}=\frac{k^{3}}{2 \pi^{2}}\left|\delta \phi_{k}\right|^{2}
$$

This can be traced back to the following general definition of a power spectrum

$$
\left\langle g_{\vec{k}} g_{\vec{k}^{\prime}}\right\rangle=\delta^{(3)}\left(\vec{k}-\vec{k}^{\prime}\right)\left|g_{k}\right|^{2} \equiv \delta^{(3)}\left(\vec{k}-\vec{k}^{\prime}\right) \frac{2 \pi^{2}}{k^{3}} \mathcal{P}_{g}(k),
$$

where $g_{k}(t)$ corresponds to the Fourier transform of some quantity $g(\vec{x}, t)$. This definition immediately gives $(3.22)$ and also implies the relation

$$
\left\langle g^{2}(\vec{x}, t)\right\rangle=\int \frac{d^{3} k}{(2 \pi)^{3}}\left|g_{k}\right|^{2}=\int d \ln k \mathcal{P}_{g}(k) .
$$

At physical scales $p=k / a$ bigger than the Hubble scale $H$ the mean square fluctuations behave as ordinary quantum fluctuations in flat space, which become negligible as one increases the length scale. However, as already explained, at physical scales $p$ smaller than the Hubble scale $H$ the field no longer oscillates, leading to large length scale fluctuations. Being interested in those scales, we use the expression for $\phi_{k}$ as $a \rightarrow \infty$ and obtain for the power spectrum, neglecting constants,

$$
\mathcal{P}_{\delta \phi} \propto H^{2+2 \lambda_{+}} k^{-2 \lambda_{+}} a^{2 \lambda_{+}} .
$$

The massless limit corresponds to taking $\lambda_{+}=0$. 
In pure de Sitter space this scalar field source will not lead to scalar curvature perturbations because the scalar field fluctuations are perfectly decoupled in that case. Another way of putting this is that in pure de Sitter these fluctuations are pure gauge. For instance consider a density perturbation $\delta \rho$, then it is easy to see that

$$
\kappa \delta \rho=\kappa \frac{d \rho}{d \phi} \delta \phi=\left(-3 H^{2} \beta\right) \delta \phi,
$$

where $\beta$ is the holographic beta-function defined in (2.24). Only when there exists a small "tilt" away from pure de Sitter space $(\beta \neq 0)$ will the scalar field fluctuations couple and induce density perturbations. To obtain the correct physical quantity denoting the scalar curvature perturbations and their relation to the scalar field fluctuations is not straightforward and we will just refer to the literature [16, 17]. We will just quote the standard result that the scalar curvature perturbation can be described by the (dimensionless) quantity

$$
\delta \mathcal{R}=\frac{\delta \rho}{p+\rho}=\left(\frac{-3 \kappa}{\beta}\right) \delta \phi,
$$

immediately leading to the following relation between the power spectra

$$
\mathcal{P}_{\mathcal{R}}=\beta^{-2} \mathcal{P}_{\delta \phi}
$$

When we will discuss this result from the deformed CFT point of view the extra factor of $\beta$ actually appears rather naturally. Note that the factor of $\beta$, for small $\beta \ll 1$, will kill the time (or scale factor) dependence of the perturbations because

$$
\beta \approx \lambda \delta \phi \Rightarrow \delta \phi \propto a^{\lambda} \Rightarrow \beta \propto \lambda a^{\lambda} .
$$

Before we go on to discuss the spectral index we should note that to connect to the standard expressions in the literature involving the slow-roll parameters $\epsilon_{H}$ and $\eta_{H}$ one uses the following definitions for the slow-roll parameters

$$
\epsilon_{H} \equiv-\frac{\partial \ln H}{\partial \ln a}, \quad \eta_{H} \equiv-\frac{\partial \ln \frac{\partial H}{\partial \phi}}{\partial \ln a},
$$

leading to the following relations between the different sets of parameters 18 : $2 \epsilon_{H}=\beta^{2}$ and $2 \eta_{H}=\beta^{2}-2 \lambda$.

We are now ready to discuss the spectral index of the scalar curvature perturbation. Since we just concluded that (3.28) is independent of time we can evaluate this expression at the time it was formed, which was estimated to be around $|\chi|=1$, so when $a=k / H$. Plugging this into the expression (3.25) we see that the $k$-dependence disappears and that we are effectively left with a power spectrum of massless perturbations. So our starting point is the following expression for the power spectrum of the scalar density perturbation

$$
\left.\mathcal{P}_{\text {scalar }} \propto\left(\frac{H}{\beta}\right)^{2}\right|_{k=a H},
$$

where we have added explicitly that this expression should be evaluated at $k=a H$. The Hubble parameter $H(a)$ and the beta-function $\beta(a)$ generically are functions of the scale 
factor $a$. Different scales $k$ "freeze" into the power spectrum at different scale factors $a(t)$ when the physical momentum $p=k / a=H$ or equivalently when $k=a H$. This effectively leads to $k$-dependence of the spectrum because $\beta$ and $H$ are functions of $a(t)$. To calculate this scale dependence we first relate derivatives with respect to $a$ to derivatives with respect to $k$

$$
\frac{d k}{d a}=\left(H+a \frac{d H}{d a}\right)=\left(1-\frac{1}{2} \beta^{2}\right) H
$$

This implies that

$$
a \frac{d}{d a}=\left(1-\frac{1}{2} \beta^{2}\right) k \frac{d}{d k} .
$$

This allows us to calculate the effective $k$-dependence of $H$ by noting that

$$
a \frac{d H}{d a}=-\frac{1}{2} \beta^{2} H=\left(1-\frac{1}{2} \beta^{2}\right) k \frac{d H}{d k} .
$$

So in the end we find the following equation for the effective $k$-dependence of $H$

$$
\frac{d \ln H}{d \ln k}=\frac{-\frac{1}{2} \beta^{2}}{\left(1-\frac{1}{2} \beta^{2}\right)} .
$$

Under the assumption that $\beta$ is approximately constant $\beta=\bar{\beta}$ (i.e. $k$-independent) and to lowest order in $\beta$ this equation is easy to solve and gives

$$
H(k) \approx k^{-\frac{1}{2} \bar{\beta}^{2}}
$$

The same procedure can be applied to find the effective $k$-dependence of $\beta$. We first note that

$$
a \frac{d \beta}{d a}=\lambda \beta=\left(1-\frac{1}{2} \beta^{2}\right) k \frac{d \beta}{d k},
$$

where we defined $\lambda$ as $\lambda \equiv d \beta / d \phi$ (instead of $\lambda \equiv \beta / \phi$ ). This implies the following equation for the effective $k$-dependence of $\beta$

$$
\frac{d \ln \beta}{d \ln k}=\frac{\lambda}{\left(1-\frac{1}{2} \beta^{2}\right)} .
$$

Again, under the assumption that $\lambda$ is approximately $k$-independent $\lambda=\bar{\lambda}$ and to lowest order in $\beta$ we find

$$
\beta(k) \approx k^{\bar{\lambda}}
$$

We are now ready to calculate the spectral index of the power spectrum given by (3.31). Typically the spectral index is defined as follows

$$
n_{s}-1 \equiv \frac{d \ln \mathcal{P}_{\text {scalar }}}{d \ln k} .
$$

Through this definition we can in fact calculate the spectral index exactly, using the expressions in the previous paragraphs. But first let us consider the situation where $\beta$ and $\lambda$ are approximately $k$-independent and concentrate on the power of $k$ in the power spectrum, 
which should naturally define the "true" spectral index, as we will discuss in a moment. From (3.36) and (3.39) we then obtain for the (average) spectral index

$$
\bar{n}_{s}-1=-\bar{\beta}^{2}-2 \bar{\lambda} \text {. }
$$

Instead using the definition (3.40) we find

$$
n_{s}-1 \equiv \frac{d \ln \mathcal{P}_{\text {scalar }}}{d \ln k}=\frac{-\beta^{2}-2 \lambda}{\left(1-\frac{1}{2} \beta^{2}\right)},
$$

which indeed corresponds to the standard expression for the spectral index in terms of the slow-roll parameters to lowest order in $\beta^{2}$; using (3.30) we reproduce $n_{s}-1=-4 \epsilon_{H}+2 \eta_{H}$.

One should realize that (3.40) will only reproduce the true spectral index when $n_{s}$ is $k$-independent. The true definition of the $k$-dependent spectral index should be $n(k)-1=$ $\ln \mathcal{P}_{\text {scalar }} / \ln k$, as is clear when writing $\mathcal{P}_{\text {scalar }} \propto k^{(n(k)-1)}$. We can find the following expression for the difference $\mathcal{E}$ between the true spectral index and the spectral index calculated with $(3.40)$

$$
\mathcal{E} \equiv n_{s}(k)-n(k)=\left(\frac{d n}{d \ln k}\right) \ln k .
$$

We can use this expression iteratively to express the true spectral index $n(k)$ in terms of an infinite derivative expansion of the other, exactly calculable, spectral index defined through $(3.40) n_{s}(k)$

$$
n(k)=n_{s}(k)-\frac{d n_{s}}{d \ln k} \ln k+\frac{d^{2} n_{s}}{(d \ln k)^{2}}(\ln k)^{2}-\cdots,
$$

where the signs alternate between odd and even powers of $\ln k$. The first derivative of $n_{s}$ with respect to $\ln k$ can be calculated exactly and gives

$$
\frac{d n_{s}}{d \ln k}=-\frac{2}{\left(1-\frac{1}{2} \beta^{2}\right)^{3}}\left(\beta^{2} \lambda(1+\lambda)+\beta\left(1-\frac{1}{2} \beta^{2}\right) \frac{d^{2} \beta}{d \phi^{2}}\right) .
$$

To lowest order one therefore finds

$$
n(k)=n_{s}(k)+2 \beta \frac{d^{2} \beta}{d \phi^{2}} \ln k+2 \beta^{2} \lambda \ln k .
$$

Clearly, when $\beta, \lambda \ll 1$ (slow-roll regime) and when $\lambda$ is not changing too rapidly or for ranges of scales $k$ that are not too large, using the definition (3.40) is not a bad approximation.

\section{Inflationary perturbations from CFT correlators}

Soon after the dS/CFT correspondence was first conjectured, it was realized that it could perhaps have interesting applications in the context of inflation [13]. That one can derive the power spectrum of inflationary perturbations from the CFT was first anticipated in [18], and was explained for massless scalar field perturbations in [19]. The results described in this section can also be found in section 6 of the recent preprint [20], which appeared during the completion of this paper. 


\subsection{Relating correlation functions to expectation values}

Before we can go on to derive the power spectrum of inflationary perturbations from deformed CFT correlators we need to know how to relate expectation values in the dS bulk to CFT correlators. This was explained in [19] and we will briefly review that result here.

CFT vacuum correlation functions can be deduced from the de Sitter bulk following the same procedure as in AdS; calculating the action and functionally differentiating with respect to the boundary fields that act as sources in the CFT (2.12). Specifically for CFT 2-pt functions of operators $\mathcal{O}$ with scaling dimension $\Delta$ this implies

$$
\left\langle\mathcal{O}_{\Delta}(x) \mathcal{O}_{\Delta}(y)\right\rangle=\left.\frac{\delta^{2} \Psi_{d S}[\phi]}{\delta \phi(x) \delta \phi(y)}\right|_{\phi=0} .
$$

At the same time it should be clear that the spectrum of scalar field fluctuations in de Sitter space that we calculated is related to the (equal time) expectation value $\left|\phi_{k}\right|^{2}=$ $\int d^{3} k^{\prime}\left\langle\phi_{\vec{k}} \phi_{\vec{k}^{\prime}}\right\rangle$. A scalar field expectation value can be expressed, up to a delta function, as a functional integral of $\phi_{k}^{2}$ weighted by the norm of the scalar field wave functional squared. The scalar field wave functional can be approximated by the classical action in de Sitter (in the semi-classical limit). This leads to the following formal expression for the expectation value of scalar field perturbations as

$$
\left\langle\phi_{\vec{k}} \phi_{-\vec{k}}\right\rangle=\int \mathcal{D} \phi \phi_{\vec{k}} \phi_{-\vec{k}}\left|\Psi_{d S}[\phi]\right|^{2} \approx \int \mathcal{D} \phi \phi^{2}\left|e^{i S_{d S}[\phi]}\right|^{2}
$$

From these expressions one can find a direct relation between de Sitter expectation values in the bulk and CFT 2-pt functions. By definition (4.1) one can formally express the wave functional (or generating functional) $\Psi_{d S}$ in terms of the 2-pt function and higher order (connected) $n$-point functions

$$
\Psi_{d S}[\phi]=e^{\frac{1}{2} \int d^{3} k d^{3} k^{\prime}\left\langle\mathcal{O}_{\vec{k}} \mathcal{O}_{\vec{k}^{\prime}}\right\rangle \phi_{\vec{k}} \phi_{\vec{k}^{\prime}}+\cdots+\frac{1}{n !} \int d^{3} k \ldots d^{3} k^{n} \phi_{\vec{k}} \ldots \phi_{\vec{k}} n\left\langle\mathcal{O}_{\vec{k}} \ldots \mathcal{O}_{\vec{k}}\right\rangle} .
$$

Plugging this expression into the expression for the expectation value (4.2), neglecting the higher order $n$-point functions, we obtain

$$
\left\langle\phi_{k} \phi_{-k}\right\rangle=\int \mathcal{D} \phi \phi^{2} e^{\int d^{3} k \int d^{3} k^{\prime} \phi_{k} \phi_{k^{\prime}} \operatorname{Re}\left\langle\mathcal{O}_{k} \mathcal{O}_{k^{\prime}}\right\rangle} .
$$

By changing the path integral variable (without changing the measure) we can isolate the dependence on the 2-pt correlation function. Defining

$$
\tilde{\phi}_{\vec{k}} \equiv i \sqrt{2 \operatorname{Re}\left\langle\mathcal{O}_{k} \mathcal{O}_{-k}\right\rangle} \phi_{\vec{k}}
$$

we obtain the following expression for the expectation value

$$
\left\langle\phi_{k} \phi_{-k}\right\rangle=\frac{-1}{2 \operatorname{Re}\left\langle\mathcal{O}_{k} \mathcal{O}_{-k}\right\rangle} \int \mathcal{D} \tilde{\phi} \tilde{\phi}^{2} e^{-\frac{1}{2} \int d^{3} k \int d^{3} k^{\prime} \tilde{\phi}_{k} \tilde{\phi}_{k^{\prime}}} .
$$

Now that we have disentangled the path integral from the correlation function it should be clear that the path integral is going to give just a number. In that case this leaves us with 
the following relation between bulk expectation values and "boundary" correlators, which was first derived in [19],

$$
\left\langle\phi_{k} \phi_{-k}\right\rangle \propto \frac{-1}{2 \operatorname{Re}\left\langle\mathcal{O}_{k} \mathcal{O}_{-k}\right\rangle} .
$$

We note that because the bulk scalar field acts as a source in the CFT, this relation is almost like a Legendre transform, except for the factor of two and the restriction to the real part of the correlators, which can respectively be traced back to the square and the norm of the scalar field wave functional in (4.2).

\subsection{CFT operators, energy momentum and inflationary perturbations}

Next we want to explicitly show that one obtains the correct expressions for the spectrum of inflationary perturbations, starting from CFT correlation functions. The vacuum 2-pt function in three euclidean dimensions of operators with arbitrary scaling dimensions $\Delta$ is given by

$$
\left\langle\mathcal{O}_{\Delta}(x) \mathcal{O}_{\Delta}\left(x^{\prime}\right)\right\rangle \propto \frac{\eta_{c}^{2 \Delta-6}}{\left|x-x^{\prime}\right|^{2 \Delta}} H^{-2},
$$

where $\eta_{c}$ corresponds to a cutoff length scale necessary to properly define the correlator. After Fourier transforming this reads

$$
\left\langle\mathcal{O}_{\Delta} \mathcal{O}_{\Delta}\right\rangle \propto H^{-2}|k|^{3}\left(\frac{\mu_{c}}{|k|}\right)^{6-2 \Delta},
$$

where $\mu_{c}=1 / \eta_{c}$ now corresponds to an energy cutoff. Note that the explicit cutoff dependence disappears when one considers marginal operators $\Delta=3$. We will write $k=|k|$ from now on.

We will use the expression (3.28) together with (3.22) to calculate the power spectrum of inflationary density perturbations. The appearance of the function $\beta$ in these expressions has a beautiful interpretation in the CFT. First note that the bulk metric acts as the source for the energy momentum tensor in the CFT (2.8). It can be shown that to discuss scalar curvature perturbations we should be interested in the trace of the energy momentum tensor [19]. In a CFT the expectation value of the trace of the energy momentum tensor vanishes. However, moving away from the conformal fixed point one finds the well known and intuitively understood expression for the conformal anomaly in terms of non-marginal CFT operators (we are only considering flat spatial backgrounds)

$$
\left\langle\operatorname{Tr} T^{i j}\right\rangle=\beta(g) \frac{\partial}{\partial g} \mathcal{L}=\beta(g)\left\langle\mathcal{O}_{\Delta}\right\rangle,
$$

where $\beta(g)$ is the beta-function for the coupling $g$ appearing in the lagrangian deforming the CFT (2.13), i.e. $\mathcal{L}=g \mathcal{O}_{\Delta}$. So we see the beta-function appearing naturally. Realizing that the expectation value of density perturbations would be proportional to the inverse of the 2-pt correlation function of the trace of the energy momentum tensor we reproduce the $\beta^{-2}$ factor in $(3.28)$. So in terms of CFT correlators the power spectrum of density fluctuations can be written as

$$
\mathcal{P}_{\mathcal{R}} \propto k^{3} \frac{1}{\operatorname{Re}\left\langle T_{i}^{i} T_{j}^{j}\right\rangle}=k^{3} \frac{1}{\beta^{2} \operatorname{Re}\left\langle\mathcal{O}_{\Delta} \mathcal{O}_{\Delta}\right\rangle} .
$$


From the general expression for the CFT correlator (4.9) it seems that we will get explicit dependence on the cutoff $\mu_{c}$ in the power spectrum. However, one should realize that $\beta(g)$ also depends on the cutoff $\mu_{c}$ as follows

$$
\beta(g) \approx(\Delta-3) g+\cdots=(\Delta-3) \mu_{c}^{\Delta-3}+\cdots .
$$

This dependence will exactly cancel the cutoff dependence of the correlation function, giving us a cutoff independent answer, as was also discussed in section 3.2. Note that this is also consistent with the fact that the energy momentum tensor in the bulk should correspond to a marginal operator in the CFT and should therefore not depend on the cutoff. The final result is

$$
\mathcal{P}_{\mathcal{R}} \propto k^{3} \frac{1}{\beta^{2} \operatorname{Re}\left\langle\mathcal{O}_{\Delta} \mathcal{O}_{\Delta}\right\rangle}=H^{2} k^{6-2 \Delta}=H^{2} k^{-2 \lambda},
$$

where $\lambda=\Delta-3=-3 / 2 \pm \sqrt{9 / 4-m^{2} / H^{2}}$. So we reproduce the expected result for the spectral index to lowest order in the departure from the fixed point (3.42) corresponding to the limit $g \rightarrow 0$, corresponding to $\beta=0$ in (3.42).

\subsection{RG-flow equations and the spectrum of inflationary perturbations}

Let us now consider adding the following deformation to the CFT action

$$
\mathcal{S}=\mathcal{S}_{\mathrm{CFT}}+g \int d^{3} x \mathcal{O}_{\Delta}
$$

This deformation will break conformal invariance and lead to RG-flow. The CFT correlators that were introduced in the previous section will now change along the RG-flow trajectory. This will therefore affect the spectrum of inflationary density perturbations as one moves further away from the conformal fixed point. To systematically follow this process one needs to study the RG-flow equations, in particular of course the Callan-Symanzik equations for the correlators. In [20] the RG-flow equations were carefully deduced from the bulk dS gravity theory (see also [23]), following an approach first applied in the AdS/CFT context by [11]. Introducing the Hamilton-Jacobi action functional in the bulk one carefully introduces counterterms for the local divergences that appear in the limit $t \rightarrow \infty$, defining an effective renormalized action. This new action functional also satisfies the Hamilton-Jacobi equation that after some rewritings and neglecting of higher order terms can be viewed as an RG equation for the renormalized action. For all the details we refer to [20, 23]. We will be interested in the evolution of the correlators, in particular the $k$ dependence along the flow that will determine the spectral index of the inflationary density perturbations.

As already explained Callan-Symanzik equations can be deduced from the bulk dS gravity theory. We will be interested in 2-pt functions $\langle\mathcal{O}(x) \mathcal{O}(y)\rangle$ only, and in that case the Callan-Symanzik equation looks as follows

$$
\left[\Lambda \frac{\partial}{\partial \Lambda}+\beta(g) \frac{\partial}{\partial g}+2 \lambda(g)\right]\left\langle\mathcal{O}_{\Delta} \mathcal{O}_{\Delta}\right\rangle=0
$$


where $\beta(g) \equiv \Lambda \frac{\partial g}{\partial \Lambda}$ with $\Lambda=\mu_{c}$ corresponding to the cutoff scale. The (anomalous) scaling dimension $\lambda(g)$ is defined as follows

$$
\lambda(g) \equiv \frac{\partial \beta}{\partial g}=\lambda+\eta(g)=\Delta-3+\eta(g),
$$

where $\lambda$ and $\Delta$ correspond to the constant scaling dimensions at a conformal fixed point (i.e. the lowest order coefficients in an expansion of $\beta(g)$ in $g$ ). Even though this definition of the scaling dimension is not the standard one, it reproduces the standard definition of non-marginal scaling dimensions in the limit of small coupling $g$. This can be seen by first noting that $g$ can be identified with the field-strength renormalization factor $g=Z^{1 / 2}$ because of the particular deformation (4.14) we are considering. Then using the more standard definition of the anomalous scaling dimension $2 \lambda(g)=\frac{\Lambda}{Z} \frac{\partial Z}{\partial \Lambda}=\frac{\beta}{g}$, we see that non-marginal scaling dimensions $\lambda \neq 0$ in the CS-equation are reproduced as the lowest order coefficients in an expansion in the coupling $g$. The higher order coefficients $a_{i}, i>1$, are related by a factor of $i$ between the two definitions.

As a consequence of straightforward dimensional analysis one can determine the cutoff dependence of a general Fourier transformed correlator as

$$
\left\langle\mathcal{O}_{\Delta} \mathcal{O}_{\Delta}\right\rangle(k)=k^{3} F\left[\frac{\Lambda^{2}}{k^{2}}, g(\Lambda)\right]
$$

which is just the generalized version of the expression (4.9) with $\Lambda=\mu_{c}$. Using this expression it is possible to rewrite the CS-equation in terms of a momentum $k$-derivative instead of the cutoff scale $\Lambda$ by noting that

$$
\Lambda \frac{\partial}{\partial \Lambda}\left\langle\mathcal{O}_{\Delta} \mathcal{O}_{\Delta}\right\rangle(k)=\left(-k \frac{\partial}{\partial k}+3\right)\langle\mathcal{O O}\rangle(k)
$$

This allows us to rewrite the CS-equation (4.15) as follows

$$
\left.\left[\frac{\partial}{\partial \ln k}-\beta(g) \frac{\partial}{\partial g}+(3-2 \Delta)-2 \eta(g)\right)\right]\left\langle\mathcal{O}_{\Delta} \mathcal{O}_{\Delta}\right\rangle=0 .
$$

The $k$-dependence of the CFT 2-pt correlators (4.9) is reproduced in the fixed point limit $g \rightarrow 0$, i.e. when one recovers exact conformal invariance so $\beta(g)=\eta(g)=0$. We will be interested in corrections to the $k$-dependence of the correlator due to RG-flow away from the fixed point. There is a quick and dirty way to see that one can reproduce the approximate expression for the spectral index (3.41). It first of all involves the assumption that the CFT correlators (4.8) only depend on the coupling $g$ through the Hubble parameter $H$, which as we will see later on is not expected to be true in general. Nevertheless proceeding, using the expression (2.26) for the beta-function and remembering that the coupling $g$ corresponds to the scalar field in the bulk, we derive that

$$
\beta(g) \frac{\partial}{\partial g}\left\langle\mathcal{O}_{\Delta} \mathcal{O}_{\Delta}\right\rangle=\beta^{2}\left\langle\mathcal{O}_{\Delta} \mathcal{O}_{\Delta}\right\rangle
$$

This relation allows one to solve the Callan-Symanzik equation (4.19) if one also assumes an approximately constant beta-function $\beta(g) \approx \bar{\beta}$ and scaling dimension $\lambda(g) \approx \bar{\lambda}=$ 
$\bar{\Delta}-3=\Delta-3+\bar{\eta}$. In that case we find that the $k$ dependence of the correlator will be modified to become

$$
\left\langle\mathcal{O}_{\Delta} \mathcal{O}_{\Delta}\right\rangle \propto k^{3} k^{\bar{\beta}^{2}+2 \bar{\Delta}-6},
$$

which will indeed reproduce the expression (3.41) for the spectral index of the power spectrum of density perturbations, using (4.11). The condition of approximately constant $\bar{\beta}$ and $\bar{\lambda}$ can be made more precise by saying that $\frac{\partial}{\partial \ln k} \beta=\beta \lambda \ll 1$ and $\frac{\partial}{\partial \ln k} \lambda=\beta \frac{\partial}{\partial g} \lambda \ll 1$, so typically these conditions can both be satisfied when $\beta \ll 1$. Although we reproduce the correct answer this way, this approach is not completely transparent and it will be useful, especially if we want to consider further corrections, to develop a more consistent method toward solving the CS-equation.

As is well-known, a more general approach toward solving the CS-equation would be to start with the following ansatz for the 2-pt correlator

$$
\left\langle\mathcal{O}_{\Delta} \mathcal{O}_{\Delta}\right\rangle \propto Z(g) k^{3+2 \lambda}
$$

separating the full $k$ and $g$ dependence. To solve the CS-equation the function $Z(g)$ is expressed as

$$
Z(g)=Z_{0} \exp \int_{0}^{g} d g^{\prime} \frac{2 \lambda-2 \lambda\left(g^{\prime}\right)}{\beta\left(g^{\prime}\right)}=Z_{0} \exp -2 \int_{\Lambda_{0}}^{\Lambda_{g}} d \ln \left(\frac{\Lambda^{\prime}}{k}\right) \eta\left(\Lambda^{\prime}\right) .
$$

One can easily check that this expression for $Z(g)$ solves the CS-equation. The expression in terms of an integral over $d \ln (\Lambda / k)$, with $k$ introduced as the only other natural scale that can appear in the correlator, has the advantage of making it possible to directly read off the $k$-dependence that we are interested in. The only thing left to do is to evaluate the function $Z(g)$ (or $Z(\Lambda / k)$ ), which for example can be done order by order in $g$. One can in fact similarly express the Hubble parameter $H^{-2}$ in terms of an integral over the coupling (or the logarithmic cutoff scale) (2.26), (2.35)

$$
H(g)^{-2}=H_{0}^{-2} \exp \int_{0}^{g} d g^{\prime} \beta\left(g^{\prime}\right)=H_{0}^{-2} \exp \int_{\Lambda_{0}}^{\Lambda_{g}} d \ln \left(\frac{\Lambda^{\prime}}{k}\right) \beta\left(\Lambda^{\prime}\right)^{2} .
$$

This allows us to write the solution $Z(g)$ as follows

$$
\begin{aligned}
Z(g) & =H(g)^{-2} \exp \int_{0}^{g} d g^{\prime} \frac{-2 \eta\left(g^{\prime}\right)-\beta\left(g^{\prime}\right)^{2}}{\beta\left(g^{\prime}\right)} \\
& =H(g)^{-2} \exp \int_{\Lambda_{0}}^{\Lambda_{g}} d \ln \left(\frac{\Lambda^{\prime}}{k}\right)\left(-2 \eta\left(\Lambda^{\prime}\right)-\beta\left(\Lambda^{\prime}\right)^{2}\right),
\end{aligned}
$$

extracting the $H^{-2}$ part from the solution $Z(g)$, with $\eta(g)$ corresponding to the anomalous part of the scaling dimension (4.16). From this general expression it is more transparent under precisely what conditions we can reproduce the spectral index of inflationary perturbations, and how to go beyond that. Isolating the Hubble parameter in $Z(g)$ means that we are interested in the induced $k$-dependence relative to the average Hubble parameter, which is exactly what is done in the bulk calculation of the inflationary power spectrum. 
The integral in the second line of (4.26) can be approximated to lowest order by assuming constant $\beta=\bar{\beta}$ and $\eta=\bar{\eta}$. Using (4.11) this immediately reproduces the expression for the spectral index of the scalar curvature perturbation we found earlier through a more "sloppy" method, agreeing with the bulk result (3.41). However, starting from (4.26) would in principle allow us to go beyond the approximation of constant $\beta$ and $\lambda$ [26]. We hope to come back to that problem in the near future.

\section{Holography and universality of inflationary perturbations}

Just as in AdS/CFT, in dS/CFT the UV and IR are interchanged with respect to bulk or boundary physics. A UV cutoff $\Lambda$ in euclidean field theory corresponds to an IR (large time/scale factor) cutoff $t_{c}$ in the bulk. The UV cutoff in the field theory implies that we will only be considering euclidean momenta $|k|<\Lambda$ and realizing that $|k|$ corresponds to the comoving momentum and that the cutoff can be written as $\Lambda=a_{c} H$ in the bulk we find the following constraint on the bulk physical momentum $|p|=|k| / a$

$$
p \leq H \text {. }
$$

The fact that this constraint on the physical momentum in the bulk only depends on the Hubble parameter tells us that dS/CFT only probes bulk physics outside the de Sitter horizon. Bulk physics inside the de Sitter horizon, as experienced by a free-falling observer, is inaccessible because it will always be integrated out in the CFT description. In terms of coarse graining it means that a static de Sitter patch always corresponds to a single lattice site in the field theory, no matter what the size of the cutoff scale in the field theory. One can in fact naturally associate an entropy to a single coarse grained region that is roughly equal to the central charge of the CFT, which is reproducing the de Sitter entropy that can be associated to a static patch of de Sitter [27], because the central charge indeed scales like the area of the static patch Hubble volume (2.36). However, the crucial reason for the entropy to scale with the area of one Hubble volume is because it corresponds to a single lattice site in the field theory. As soon as one considers larger physical bulk volumes containing more than a single lattice site one will find that the entropy will scale with the total three-dimensional volume, instead of the area, as expected from a three-dimensional field theory. What dS/CFT does for us is to identify a single Hubble volume as the unique volume at which the entropy indeed scales like the area, corresponding to a single lattice site in the field theory. This is all dS/CFT will ever have to say about holography in the static patch, because physics inside the static patch can not be probed by dS/CFT. One can therefore question the validity of objections raised against dS/CFT that rely on physics in the de Sitter static patch [7]. The applicability, if any, of dS/CFT should instead be on bulk length scales larger than the Hubble radius. This is in fact just right for a treatment of the spectrum of inflationary perturbations using CFT correlators, which is only formed after wavelengths cross the Hubble radius. This is of course what we have shown, that one can calculate the spectrum of inflationary perturbations using deformed CFT correlators.

In the previous paragraph we pointed out that, according to dS/CFT, the holographic area-entropy relation immediately breaks down on length scales larger than the Hubble 
radius and the ordinary scaling of entropy with the total volume is found instead. We therefore conclude that even though dS/CFT is considered to be a "holographic" correspondence, it does not predict any holographic constraints (having to do with the area law for the gravitational entropy) on the spectrum of inflationary perturbations. Similar ideas in support of the (near) absence of holographic constraints on the CMB spectrum have been described in 28], however opposite claims have also been reported [29]. It would perhaps be interesting to study this in more detail. This slightly counter-intuitive result is due to the fact that in dS/CFT the "holographic" coordinate is timelike and holography therefore acquires a completely different meaning, as we will now discuss.

The usual bulk understanding of inflationary perturbations is as originating from vacuum fluctuations that grow to become the size of the Hubble horizon after which they freeze and become part of the spectrum of inflationary perturbations. This understanding does not immediately explain why the spectrum of inflationary perturbations seems to be so universal, i.e. very independent of microscopic physics. In this new approach based on deformed CFT correlators and RG-flow equations the static patch is completely removed (or coarse grained) and nevertheless we still recover the correct spectrum of inflationary perturbations. As already explained the static patch, corresponding to a single Hubble volume, corresponds to scales above the cutoff from the deformed CFT point of view. This necessarily implies that the spectrum of inflationary perturbations is very independent of the physics at bulk (length) scales smaller than the horizon, except for its effect on certain universal parameters (homogeneous fields in the bulk and couplings in the deformed CFT), in exactly the spirit of the Wilsonian renormalization group. It is this concept of universality that should be the main message of (timelike) holography. The deformed CFT approach explicitly realizes the strong independence of the spectrum of inflationary perturbations from detailed sub-horizon or trans-Planckian dynamics. So one advantage of the CFT approach is that it makes the universal nature of the primordial CMB spectrum explicit. Note that the primordial CMB spectrum can and will of course still depend on initial (boundary) conditions that one imposes on sub- and/or super-horizon scales. In the quantum theory this corresponds to selecting a different vacuum which will affect the spectrum, a fact that has attracted a lot of attention recently [30]. From the CFT point of view, changing boundary conditions or vacua in the bulk will affect the bulk action and therefore the CFT correlators, see also [31]. It would perhaps be interesting to study this in more detail. We hope to come back to these and other matters in the near future.

\section{Note added}

During the final stages of this work [20] appeared in which essentially the same ideas are described. In particular our paper has a lot of overlap with section 6 in their paper. We have tried to highlight the dS/CFT point of view and commented on holography and universality in the context of the power spectrum of inflationary perturbations. Instead of our emphasis on a UV field theoretic point of view, the authors of [20] focus more on the complementary bulk gravitational IR point of view, which is very interesting in its own right. 


\section{Acknowledgments}

I would like to thank F. Larsen, R. Leigh, D. Schwarz, D. Chung, A. Petkou, D. Kabat, D. Minic, K. Schalm and G. Shiu for many interesting and useful discussions. I thank K. Schalm for reading a first draft and suggesting improvements. The Amsterdam Summer Workshop is acknowledged for hospitality and for providing an inspiring atmosphere.

\section{References}

[1] C.L. Bennett et al., First year wilkinson microwave anisotropy probe (WMAP) observations: preliminary maps and basic results, Astrophys. J. Suppl. 148 (2003) 1 astro-ph/0302207.

[2] Supernova Search Team collaboration, A.G. Riess et al., Observational evidence from supernovae for an accelerating universe and a cosmological constant, Astron. J. 116 (1998) 1009-1038 astro-ph/9805201;

Supernova Cosmology Project collaboration, S. Perlmutter et al., Measurements of omega and lambda from 42 high-redshift supernovae, Astrophys. J. 517 (1999) 565 astro-ph/9812133.

[3] G. 't Hooft, Dimensional reduction in quantum gravity, gr-qc/9310026;

L. Susskind, The world as a hologram, J. Math. Phys. 36 (1995) 6377 hep-th/9409089.

[4] J.M. Maldacena, The large- $N$ limit of superconformal field theories and supergravity, Adv. Theor. Math. Phys. 2 (1998) 231 hep-th/9711200.

[5] O. Aharony, S.S. Gubser, J.M. Maldacena, H. Ooguri and Y. Oz, Large-N field theories, string theory and gravity, Phys. Rept. 323 (2000) 183 hep-th/9905111.

[6] A. Strominger, The dS/CFT correspondence, J. High Energy Phys. 10 (2001) 034 hep-th/0106113;

C.M. Hull, Timelike t-duality, de Sitter space, large- $N$ gauge theories and topological field theory, J. High Energy Phys. 07 (1998) 021 hep-th/9806146.

[7] N. Goheer, M. Kleban and L. Susskind, The trouble with de Sitter space, J. High Energy Phys. 07 (2003) 056 hep-th/0212209;

L. Dyson, M. Kleban and L. Susskind, Disturbing implications of a cosmological constant, J. High Energy Phys. 10 (2002) 011 [hep-th/0208013]; Is there really a de Sitter/CFT duality, J. High Energy Phys. 08 (2002) 045 hep-th/0202163.

[8] M. Porrati and A. Starinets, Rg fixed points in supergravity duals of 4-d field theory and asymptotically AdS spaces, Phys. Lett. B 454 (1999) 77 hep-th/9903085.

[9] V. Balasubramanian and P. Kraus, Spacetime and the holographic renormalization group, Phys. Rev. Lett. 83 (1999) 3605 hep-th/9903190.

[10] D.Z. Freedman, S.S. Gubser, K. Pilch and N.P. Warner, Renormalization group flows from holography supersymmetry and a C-theorem, Adv. Theor. Math. Phys. 3 (1999) 363 hep-th/9904017.

[11] J. de Boer, E. Verlinde and H. Verlinde, On the holographic renormalization group, J. High Energy Phys. 08 (2000) 003 hep-th/9912012. 
[12] M. Bianchi, D.Z. Freedman and K. Skenderis, Holographic renormalization, Nucl. Phys. B 631 (2002) 159 hep-th/0112119; How to go with an RG flow, J. High Energy Phys. 08 (2001) 041 hep-th/0105276;

K. Skenderis, Lecture notes on holographic renormalization, Class. and Quant. Grav. 19 (2002) 5849 hep-th/0209067.

[13] A. Strominger, Inflation and the $d S / C F T$ correspondence, J. High Energy Phys. 11 (2001) 049 hep-th/0110087.

[14] V. Balasubramanian, J. de Boer and D. Minic, Mass, entropy and holography in asymptotically de Sitter spaces, Phys. Rev. D 65 (2002) 123508 hep-th/0110108.

[15] V.F. Mukhanov and G.V. Chibisov, Quantum fluctuation and 'nonsingular' universe, Sov. Phys. JETP Lett. 33 (1981) 532; The vacuum energy and large scale structure of the universe, Sov. Phys. JETP 56 (1982) 258;

A.A. Starobinsky, Dynamics of phase transition in the new inflationary universe scenario and generation of perturbations, Phys. Lett. B 117 (1982) 175;

A.H. Guth and S.Y. Pi, Fluctuations in the new inflationary universe, Phys. Rev. Lett. 49 (1982) 1110. J.M. Bardeen, P.J. Steinhardt and M.S. Turner, Spontaneous creation of almost scale - free density perturbations in an inflationary universe, Phys. Rev. D 28 (1983) 679.

[16] V.F. Mukhanov, H.A. Feldman and R.H. Brandenberger, Theory of cosmological perturbations. Part 1. Classical perturbations. Part 2. Quantum theory of perturbations. Part 3. Extensions, Phys. Rept. 215 (1992) 203.

[17] A. Liddle and D. Lyth, Cosmological inflation and large scale structure, Cambridge University Press, Cambridge 2000.

[18] F. Larsen, J.P. van der Schaar and R.G. Leigh, de Sitter holography and the cosmic microwave background, J. High Energy Phys. 04 (2002) 047 hep-th/0202127.

[19] J. Maldacena, Non-gaussian features of primordial fluctuations in single field inflationary models, J. High Energy Phys. 05 (2003) 013 astro-ph/0210603.

[20] F. Larsen and R. McNees, Inflation and de Sitter holography, J. High Energy Phys. 07 (2003) 051 hep-th/0307026.

[21] N. Kaloper, M. Kleban, A.E. Lawrence and S. Shenker, Signatures of short distance physics in the cosmic microwave background, Phys. Rev. D 66 (2002) 123510 hep-th/0201158;

U.H. Danielsson, A note on inflation and transplanckian physics, Phys. Rev. D 66 (2002) 023511 hep-th/0203198;

R. Easther, B.R. Greene, W.H. Kinney and G. Shiu, A generic estimate of trans-planckian modifications to the primordial power spectrum in inflation, Phys. Rev. D 66 (2002) 023518 hep-th/0204129;

C.P. Burgess, J. Cline, F. Lemieux and R. Holman, Decoupling, trans-planckia and inflation, astro-ph/0306236;

N. Kaloper and M. Kaplinghat, Primeval corrections to the CMB anisotropies, Phys. Rev. D 68 (2003) 123522 hep-th/0307016.

[22] F. Leblond, D. Marolf and R.C. Myers, Tall tales from de Sitter space. II: Field theory dualities, J. High Energy Phys. 01 (2003) 003 [hep-th/0211025]; Tall tales from de Sitter space. I: Renormalization group flows, J. High Energy Phys. 06 (2002) 052 hep-th/0202094; R. Argurio, Comments on cosmological RG flows, J. High Energy Phys. 12 (2002) 057 hep-th/0202183; 
K.R. Kristjansson and L. Thorlacius, Cosmological models and renormalization group flow, . High Energy Phys. 05 (2002) 011 hep-th/0204058.

[23] S. Nojiri and S.D. Odintsov, Conformal anomaly from dS/CFT correspondence, Phys. Lett. B 519 (2001) 145 hep-th/0106191; Asymptotically de Sitter dilatonic space-time, holographic $R G$ flow and conformal anomaly from (dilatonic) dS/CFT correspondence, Phys. Lett. B 531 (2002) 143 hep-th/0201210.

[24] E. Witten, Anti-de Sitter space and holography, Adv. Theor. Math. Phys. 2 (1998) 253 hep-th/9802150;

S.S. Gubser, I.R. Klebanov and A.M. Polyakov, Gauge theory correlators from non-critical string theory, Phys. Lett. B 428 (1998) 105 hep-th/9802109.

[25] D.S. Salopek and J.R. Bond, Nonlinear evolution of long wavelength metric fluctuations in inflationary models, Phys. Rev. D 42 (1990) 3936.

[26] W.H. Kinney, Inflation: flow, fixed points and observables to arbitrary order in slow roll, Phys. Rev. D 66 (2002) 083508 astro-ph/0206032.

[27] D. Kabat and G. Lifschytz, De Sitter entropy from conformal field theory, J. High Energy Phys. 04 (2002) 019 hep-th/0203083.

[28] U.H. Danielsson, Inflation, holography and the choice of vacuum in de Sitter space, J. High Energy Phys. 07 (2002) 040 hep-th/0205227;

Holography, inflation and quantum fluctuations in the early universe, JCAP 03 (2003) 002 hep-th/0301182.

[29] A. Albrecht, N. Kaloper and Y.-S. Song, Holographic limitations of the effective field theory of inflation, hep-th/0211221.

E. Keski-Vakkuri and M.S. Sloth, Holographic bounds on the uv cutoff scale in inflationary cosmology, JCAP 08 (2003) 001 hep-th/0306070].

[30] T. Banks and L. Mannelli, de Sitter vacua, renormalization and locality, Phys. Rev. D 67 (2003) 065009 hep-th/0209113;

N. Kaloper, M. Kleban, A. Lawrence, S. Shenker and L. Susskind, Initial conditions for inflation, J. High Energy Phys. 11 (2002) 037 hep-th/0209231;

U.H. Danielsson, On the consistency of de Sitter vacua, J. High Energy Phys. 12 (2002) 025 hep-th/0210058;

K. Goldstein and D.A. Lowe, A note on alpha-vacua and interacting field theory in de Sitter space, Nucl. Phys. B 669 (2003) 325 hep-th/0302050;

G.L. Alberghi, R. Casadio and A. Tronconi, Trans-planckian footprints in inflationary cosmology, Phys. Lett. B 579 (2004) 1 gr-qc/0303035;

C. Armendariz-Picon and E.A. Lim, Vacuum choices and the predictions of inflation, JCAP $12(2003) 006$ hep-th/0303103;

M.B. Einhorn and F. Larsen, Squeezed states in the de Sitter vacuum, Phys. Rev. D 68 (2003) 064002 hep-th/0305056;

H. Collins, R. Holman and M.R. Martin, The fate of the alpha-vacuum, Phys. Rev. D 68 (2003) 124012 hep-th/0306028.

[31] R. Bousso, A. Maloney and A. Strominger, Conformal vacua and entropy in de Sitter space, Phys. Rev. D 65 (2002) 104039 hep-th/0112218;

M. Spradlin and A. Volovich, Vacuum states and the S-matrix in dS/CFT, Phys. Rev. D 65 (2002) 104037 hep-th/0112223. 